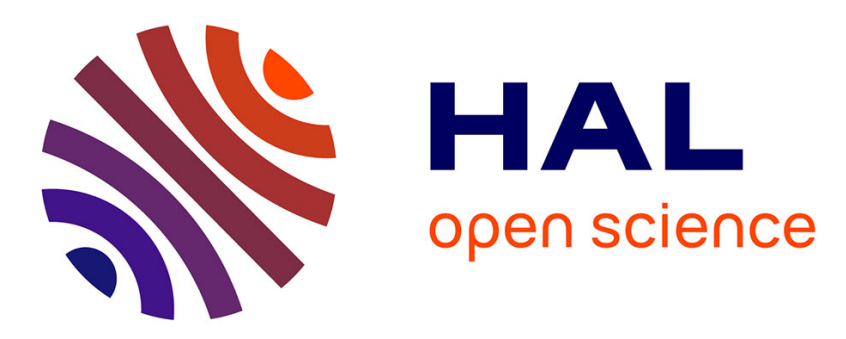

\title{
Theoretical description of two-photon phase conjugation in polar molecules
}

\author{
Claudine Hoerner, Jean-Pascal Lavoine, Albert A. Villaeys
}

\section{To cite this version:}

Claudine Hoerner, Jean-Pascal Lavoine, Albert A. Villaeys. Theoretical description of two-photon phase conjugation in polar molecules. Physical Review A: Atomic, molecular, and optical physics [1990-2015], 1993, 48 (2), pp.1564-1572. 10.1103/PhysRevA.48.1564 . hal-00880762

\section{HAL Id: hal-00880762 https://hal.science/hal-00880762}

Submitted on 7 Nov 2013

HAL is a multi-disciplinary open access archive for the deposit and dissemination of scientific research documents, whether they are published or not. The documents may come from teaching and research institutions in France or abroad, or from public or private research centers.
L'archive ouverte pluridisciplinaire HAL, est destinée au dépôt et à la diffusion de documents scientifiques de niveau recherche, publiés ou non, émanant des établissements d'enseignement et de recherche français ou étrangers, des laboratoires publics ou privés. 


\title{
Theoretical description of two-photon phase conjugation in polar molecules
}

\author{
C. Hoerner, J. P. Lavoine, and A. A. Villaeys \\ Institut de Physique et Chimie des Materiaux de Strasbourg, Groupe d'Optique Nonlinéaire et d'Optólectronique, \\ 5, rue de l' Université, 67084 Strasbourg CEDEX, France
}

(Received 30 November 1992)

\begin{abstract}
Degenerate four-wave-mixing phase conjugation is analyzed in the case of two-photon transitions between levels having unequal dipole moments. The theoretical treatment is based upon a nonperturbative solution of the density matrix and does not require the rotating-wave approximation. We give an analytical expression for the polarization component oscillating at the field frequency for a medium excited by stationary waves. In the weak-probe-beam limit, the contributions in the direction of the probe and conjugated beams are evaluated. For instance, by using this two-photon process, instead of the usual singlephoton one which is independent of the permanent dipole moments even in the strong-field limit, it is shown that lower intensities are sufficient to reach higher reflectivities. The influences of the permanent dipole moments, relaxation rates, and field frequency are also discussed.
\end{abstract}

PACS number(s): 42.65.Hw

\section{INTRODUCTION}

The generation of phase-conjugated waves by degenerate four-wave mixing (DFWM) has been the subject of intense experimental and theoretical investigations during the last decade $[1-20]$. Not only the generation but amplification and even oscillation of the conjugated fields have been predicted and observed experimentally [4-7]. For instance, a few years ago Abrams and Lind did an extensive treatment of a two-level system interacting with two counterpropagating pump waves, one probe wave, and the resulting conjugated wave, all having the same frequency [8]. In their description, the pump waves can be intense enough to saturate the medium, while the dependence of the polarization on the probe and conjugate waves is restricted to the linear regime. Their theory has been generalized by Fu and Sargent to the nondegenerate case. Then the signal-wave frequency can deviate from the pump-wave frequency [9]. Later, Brown included the effects of pump absorption and depletion [10]. More recently, further studies have been developed to include processes such as saturation effects in Dopplerbroadened media [11] or transient phenomena [12-15]. Finally, optical phase conjugation by two-photon DFWM has also been the subject of many works [16-20].

In this paper, we analyze a two-photon phaseconjugation process in a medium modeled by two-level systems having unequal dipole moments. It has been shown previously that such systems exhibit multiphoton transitions [21,22], enhanced two-photon transitions $[23,24]$, second-harmonic generation [25], Raman scattering and wave mixing of arbitrary order [26], and optical bistability $[27,28]$. The effects of permanent dipole moments on single-photon and multiphoton resonance profiles of two-level systems interacting with either a monochromatic field, two nondegenerate optical fields, an optical field applied simultaneously with a static electric field, or a short pulse in the presence of a continuous wave have also been considered in the literature [29-36].
Recently, Lavoine, Hoerner, and Villaeys have calculated the third-order nonlinear polarization of a homogeneously broadened two-level system with permanent dipole moments [37]. The role of the permanent dipole moments, as well as the influence of the rotational diffusion and field polarizations on a two-photon DFWM process, has been studied. Moreover, this process has also been discussed in the particular case of phase conjugation [38]. However, only the weak-field-limit case has been described by using a perturbative approach. Therefore it does not include the case where large reflectivities are achieved because relatively high intensities are involved.

The purpose of this work is to analyze DFWM phase conjugation in a medium modeled by two-level systems having unequal permanent dipole moments under strong excitation. In Sec. II, the density matrix formalism is introduced to establish the expression of the polarization oscillating at the field frequency. Assuming that all fields have the same frequency and parallel polarization, the Liouville equation has been solved in the steady-state regime. Our treatment does not require either the rotating-wave approximation (RWA) or a perturbational treatment. Of particular interest is the inclusion of the off-resonance polarization terms because the process under study occurs far from the resonance frequency. We shall assume that the molecules in the medium are parallel. This assumption is not very restrictive since in the perturbative limit it has been shown that for large dephasing rates rotational diffusion has no effect on such two-photon processes [37]. This softens the limitation of the approximation. In Sec. III, we evaluate the powerreflection coefficient and, in Sec. IV, we discuss the influence of the frequency detuning, dephasing rates, and permanent dipole moments on this coefficient, especially near the threshold intensity at which coupled-mode oscillations are predicted. Finally, by using this two-photon process, it is clearly demonstrated that high reflectivities can be reached very often for lower light intensities than those required by the one-photon process described by 
Abrams and Lind. A criterion which states this interesting situation is established.

\section{THEORETICAL FORMULATION}

The dynamical evolution of a quantum system undergoing relaxation and dephasing processes, and which is optically excited, is well described by the Liouville equation which takes the form

$$
\frac{\partial \rho}{\partial t}=-\frac{i}{\hbar}\left[H_{0}+V, \rho\right]-\Gamma \rho
$$

Here, $\rho(t)$ is the density matrix of the total system, $H_{0}$ the unperturbed Hamiltonian of the system alone,

$$
\left(H_{0}\right)_{i j}=\hbar \omega_{i j} \delta_{i j},
$$

$\mu$ the dipole moment, and

$$
\boldsymbol{V}=-\boldsymbol{\mu} \cdot \mathbf{E}
$$

the dipole radiation-matter interaction generated by the classical optical field $\mathbf{E}$. As usual, $\Gamma$ is the tetradic damping operator. In the Markov approximation, we only need to consider $\Gamma_{i i i i}$ which is the total decay rate of level $i, \Gamma_{i i j j}$, the transition rate of the $j \rightarrow i$ transition, and the dephasing constant

$$
\Gamma_{i j i j}=\frac{1}{2}\left(\Gamma_{i i i i}+\Gamma_{j j j j}\right)+\Gamma_{i j i j}^{(d)},
$$

where $\Gamma_{i j i j}^{(d)}$ stands for the pure dephasing constant. These matrix elements satisfy the sum rule

$$
\Gamma_{i i i i}=-\sum_{j(\neq i)} \Gamma_{j j i i} .
$$

The active medium is modeled by an ensemble of homogeneously broadened two-level systems. We label 1 the ground state and 2 the excited state. Each two-level system is characterized by longitudinal and transverse decay rates

$$
\Gamma_{1}=\Gamma_{1111}+\Gamma_{2222}
$$

and

$$
\Gamma_{2}=\Gamma_{1212}=\Gamma_{2121},
$$

respectively. The transition energy is defined by $\hbar \omega_{21}=\hbar\left(\omega_{2}-\omega_{1}\right)$. We assume that the energy levels of the microscopic two-level systems have permanent dipole moments so that $\boldsymbol{\mu}$ have nondiagonal and diagonal components, as well. They can be written as

$$
\boldsymbol{\mu}=\left[\begin{array}{ll}
\boldsymbol{\mu}_{11} & \boldsymbol{\mu}_{12} \\
\boldsymbol{\mu}_{21} & \boldsymbol{\mu}_{22}
\end{array}\right] \text {. }
$$

Consider the situation illustrated in Fig. 1. The stationary electric field is taken as

$$
\mathbf{E}=\mathscr{E} e^{i \omega t}+\text { c.c. , }
$$

with

$$
\mathscr{E}=\sum_{j} \mathscr{E}_{j} e^{-i \mathbf{k}_{j} \cdot \mathbf{r}}, j=f, b, p, \text { and } s,
$$

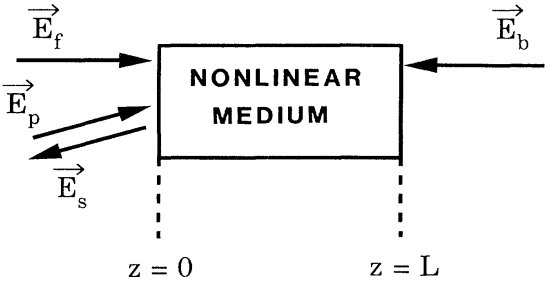

FIG. 1. Representation of the four-wave-mixing configuration used in the present study. $\mathscr{E}_{p}$ and $\mathscr{E}_{s}$ are the nonsaturating probe and conjugate waves, respectively.

where the subscripts $f$ and $b$ denote the forward- and backward-directed pump waves and $p$ and $s$ the probe and reflected signal waves. The $\mathbf{k}$-vector phase-matching conditions for DFWM are

$$
\mathbf{k}_{f}+\mathbf{k}_{b}-\mathbf{k}_{p}-\mathbf{k}_{s}=\mathbf{0} \text {. }
$$

All fields are assumed to have parallel polarizations and the angle between $\mathbf{k}_{f}$ and $\mathbf{k}_{p}$ is assumed to be small. To simplify our model, we consider $\boldsymbol{\mu}$ as a real matrix and all dipole moments parallel to the field polarization. This last assumption is not restrictive, most molecules having lower than $10^{\circ}$ angles between the various dipole moments. Therefore vector notation can be omitted.

The microscopic polarization $P$ of a two-level system whose levels have permanent dipole moments is given by

$$
\begin{aligned}
& P=\operatorname{Tr}(\mu \rho), \\
& P=\mu_{12}\left(\rho_{21}+\rho_{12}\right)+\frac{d}{2} w .
\end{aligned}
$$

Here, $w=\rho_{22}-\rho_{11}$ stands for the population difference and is equal to $w_{s}$ in the absence of fields,

$$
w_{s}=\frac{\Gamma_{1111}-\Gamma_{2222}}{\Gamma_{1111}+\Gamma_{2222}} \text {. }
$$

The quantity $d$ is the difference between the permanent dipole moments of the ground and excited states, that is to say, $d=\mu_{22}-\mu_{11}$. In terms of population difference and coherence, the Liouville equation takes the form

$$
\begin{aligned}
& \frac{\partial w}{\partial t}=\frac{-2 i \mu_{12}}{\hbar} E\left(\rho_{21}-\rho_{12}\right)-\Gamma_{1}\left(w-w_{s}\right) \\
& \frac{\partial \rho_{12}}{\partial t}=\left(i \omega_{21}-\Gamma_{2}\right) \rho_{12}+\frac{i \mu_{12}}{\hbar} E w-\frac{i d}{\hbar} E \rho_{12} \\
& \rho_{21}=\rho_{12}^{*} .
\end{aligned}
$$

The nonlinear coupling between field and system leads to harmonic generation. Therefore the density matrix elements can be expanded in a Fourier series of the form [24]

$$
\rho_{i j}=\sum_{m} \rho_{i j}^{(m)} e^{i m \omega t},
$$

where $\rho_{i j}^{(m)}$ are slowly varying terms. Substituting expansion (2.14) along with the field (2.8) into the density matrix equation (2.13) leads to recursion relations between the coefficients $\rho_{i j}^{(m)}$. These recursion relations are tedi- 

cated to retain only the components of lowest order on field amplitude up to $m=2$. Consequently, expression (2.14) reduces to

$$
\begin{aligned}
& w=w_{0}+w_{1} e^{i \omega t}+w_{2} e^{-i \omega t}+w_{3} e^{2 i \omega t}+w_{4} e^{-2 i \omega t}, \\
& \rho_{12}=\rho_{0}+\rho_{1} e^{i \omega t}+\rho_{2} e^{-i \omega t}+\rho_{3} e^{2 i \omega t}+\rho_{4} e^{-2 i \omega t} .
\end{aligned}
$$

This treatment is nonperturbative because all the couplings between all the density matrix elements are included exactly. In particular, one- and two-photon processes are included to infinite order. However, this procedure rejects the rapidly oscillating terms which correspond to third- or higher-order processes, so that we exclude net three- and higher-photon absorption.

Furthermore, for our purpose, we have to include in the model multiphoton processes like those described in Fig. 2. This figure shows clearly that the only terms $\rho_{i j}^{(m)}$ which lead to important contributions are those having the same frequency dependence as the terms appearing in the perturbative limit: $w_{0}$ and $\rho_{1}$ near the one-photon resonance and $w_{0}, w_{1}, w_{2}, \rho_{1}$, and $\rho_{3}$ near the two-photon resonance. Notice that the dots stand for an arbitrary number of pairs of absorbed and emitted photons which are processes having net zero-photon absorption. Therefore expansion (2.15) reduces to

Also, because $w$ is a real quantity we have $w_{2}=w_{1}^{*}$. Substitution of expansion (2.16) into Eqs. (2.13) yields

$$
i \frac{\partial \rho}{\partial t}=\underline{A} \rho+\underline{B}
$$

where ous. For the sake of simplicity, the Fourier series is trun-

$$
\begin{aligned}
& w=w_{0}+w_{1} e^{i \omega t}+w_{2} e^{-i \omega t}, \\
& \rho_{12}=\rho_{1} e^{i \omega t}+\rho_{3} e^{2 i \omega t} .
\end{aligned}
$$

(a)

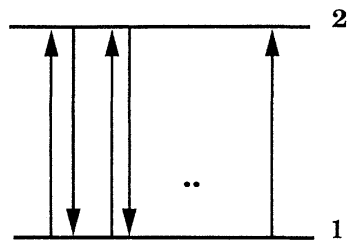

(b)

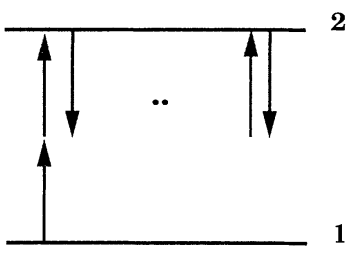

FIG. 2. We sketch the diagrams associated with multiphoton processes having net one-photon absorption for (a) $\omega \simeq \omega_{21}$ and (b) $\omega \simeq \omega_{21} / 2$. The dots symbolize an arbitrary number of pairs of absorbed and emitted photons. In both cases, the three first arrows represent the diagrams associated with the perturbative limit. In our model these processes are included to infinite order.

$$
\rho=\left(\begin{array}{c}
w_{0} \\
w_{1} \\
w_{2} \\
\rho_{1} \\
\rho_{3} \\
\rho_{1}^{*} \\
\rho_{3}^{*}
\end{array}\right), \underline{B}=\left(\begin{array}{c}
-G_{1} w_{s} \\
0 \\
0 \\
0 \\
0 \\
0 \\
0
\end{array}\right)
$$

and

$$
\underline{A}=\left(\begin{array}{ccccccc}
G_{1} & 0 & 0 & -2 X \mathscr{E}^{*} & 0 & 2 X \mathscr{E} & 0 \\
0 & G_{2} & 0 & 0 & -2 X \mathscr{E}^{*} & 0 & 0 \\
0 & 0 & G_{3} & 0 & 0 & 0 & 2 X \mathscr{E} \\
-X \mathscr{E} & 0 & 0 & G_{7} & Y \mathscr{E}^{*} & 0 & 0 \\
0 & -X \mathscr{E} & 0 & Y \mathscr{E} & G_{9} & 0 & 0 \\
X \mathscr{E}^{*} & 0 & 0 & 0 & 0 & G_{12} & -Y \mathscr{E} \\
0 & 0 & X \mathscr{E}^{*} & 0 & 0 & -Y \mathscr{E}^{*} & G_{14}
\end{array}\right),
$$

with

$$
\begin{aligned}
& X=\frac{\mu_{12}}{2 \hbar}, \quad Y=\frac{d}{2 \hbar}, \quad G_{1}=-i \Gamma_{1}, \quad G_{2}=-i \Gamma_{1}+\omega, \quad G_{3}=-i \Gamma_{1}-\omega, \\
& G_{7}=-i \Gamma_{2}-\omega_{21}+\omega, \quad G_{9}=-i \Gamma_{2}-\omega_{21}+2 \omega, \quad G_{12}=-G_{7}^{*}, \text { and } G_{14}=-G_{9}^{*} .
\end{aligned}
$$

All previous notations will be useful to simplify the evaluation of the population and the coherences created in the system. 


\section{EVALUATION OF THE POWER-REFLECTION COEFFICIENT}

The evaluation of the power-reflection coefficient requires the solution of Eq. (2.17). In the steady-state regime, it can be obtained easily. Therefore the components of the polarization oscillating at the field frequency

$$
\mathcal{P}(\omega)=\mu_{12} \rho_{1}+\frac{d}{2} w_{1}
$$

can be deduced. It takes the form

$$
\mathcal{P}(\omega)=-i \hbar w_{s} \Gamma_{1} X^{2} \mathscr{E} \frac{\left[|\mathscr{E}|^{2}\left(2 X^{2} G_{12}+Y^{2} G_{3}\right)-G_{14} G_{12} G_{3}\right]\left[|\mathscr{E}|^{2}\left(2 X^{2}+Y^{2}\right)-G_{9} G_{2}\right]}{A_{0}|\mathscr{E}|^{6}+A_{1}|\mathscr{E}|^{4}+A_{2}|\mathscr{E}|^{2}+A_{3}},
$$

where

$$
\begin{aligned}
A_{0}= & -4 X^{4}\left[2 X^{2}\left(G_{12}+G_{7}\right)+Y^{2}\left(G_{3}+G_{2}\right)\right] \\
A_{1}= & 4 X^{4}\left(G_{14} G_{12} G_{3}+G_{14} G_{7} G_{3}+G_{12} G_{9} G_{2}\right. \\
& \left.+G_{12} G_{7} G_{1}+G_{9} G_{7} G_{2}\right) \\
& +2 X^{2} Y^{2}\left(G_{14} G_{3} G_{2}+G_{12} G_{2} G_{1}+G_{9} G_{3} G_{2}\right. \\
& \left.\quad+G_{7} G_{3} G_{1}\right)+Y^{4} G_{3} G_{2} G_{1}, \\
A_{2}= & -2 X^{2}\left(G_{14} G_{12} G_{9} G_{3} G_{2}+G_{14} G_{12} G_{7} G_{3} G_{1}\right. \\
& \left.\quad+G_{14} G_{9} G_{7} G_{3} G_{2}+G_{12} G_{9} G_{7} G_{2} G_{1}\right) \\
& -Y^{2}\left(G_{14} G_{12} G_{3} G_{2} G_{1}+G_{9} G_{7} G_{3} G_{2} G_{1}\right) \\
A_{3}= & G_{14} G_{12} G_{9} G_{7} G_{3} G_{2} G_{1} .
\end{aligned}
$$

This polarization characterizes the response of the medium, oscillating at the field pulsation, for DFWM in the presence of arbitrary strong fields. For nonpolar molecules, $Y=0$ and we recover the well-known result obtained in the near-resonant case [39]. In general, when $Y \neq 0, P(\omega)$ can be written into the form

$\mathcal{P}(\omega)=C(\omega) \frac{\mathscr{E}\left(|\mathscr{E}|^{2}-y_{0}\right)\left(|\mathscr{E}|^{2}-y_{1}\right)}{\left(|\mathscr{E}|^{2}-x_{1}\right)\left(|\mathscr{E}|^{2}-x_{2}\right)\left(|\mathscr{E}|^{2}-x_{3}\right)}$, with

$$
\begin{aligned}
& C(\omega)=-i \hbar w_{s} \Gamma_{1} \frac{\left(2 X^{2}+Y^{2}\right)\left(2 X^{2} G_{12}-Y^{2} G_{3}\right)}{8 X^{2}\left(2 \Gamma_{2} X^{2}+\Gamma_{1} Y^{2}\right)}, \\
& y_{0}=\frac{G_{9} G_{2}}{\left(2 X^{2}+Y^{2}\right)}, \quad y_{1}=\frac{-G_{14} G_{12} G_{3}}{\left(2 X^{2} G_{12}-Y^{2} G_{3}\right)},
\end{aligned}
$$

the quantities $x_{1}, x_{2}, x_{3}$ being the roots of the equation

$$
A_{0} x^{3}+A_{1} x^{2}+A_{2} x+A_{3}=0 \text {. }
$$

In the weak-probe limit, it is assumed that the probeand signal-wave amplitudes are small compared with those of the pump waves. In that case, $P(\omega)$ can be expanded to first order in $\mathscr{E}_{s}+\mathscr{E}_{p}$. By taking

$$
\mathscr{E}=\mathscr{E}_{0}+\Delta \mathscr{E}
$$

with

$$
\mathscr{E}_{0}=\mathscr{E}_{f} e^{-i \mathbf{k}_{f} \cdot \mathbf{r}}+\mathscr{E}_{b} e^{-i \mathbf{k}_{b} \cdot \mathbf{r}}
$$

and

$$
\Delta \mathscr{E}=\mathscr{E}_{s} e^{-i \mathbf{k}_{s} \cdot \mathbf{r}}+\mathscr{E}_{p} e^{-i \mathbf{k}_{p} \cdot \mathbf{r}},
$$

we can expand $\mathcal{P}(\omega)$ as

$$
\mathcal{P}(\omega)=\mathscr{E}_{0} \mathscr{P}_{1}(\omega)+\Delta \mathscr{E} \mathcal{P}_{2}(\omega)+\Delta \mathscr{E}^{*} \mathcal{P}_{3}(\omega)
$$

Here, we have

$$
\begin{aligned}
& \mathscr{P}_{1}(\omega)=C(\omega) \frac{\left(\left|\mathscr{E}_{0}\right|^{2}-y_{0}\right)\left(\left|\mathscr{E}_{0}\right|^{2}-y_{1}\right)}{\left(\left|\mathscr{E}_{0}\right|^{2}-x_{1}\right)\left(\left|\mathscr{E}_{0}\right|^{2}-x_{2}\right)\left(\left|\mathscr{E}_{0}\right|^{2}-x_{3}\right)}, \\
& \mathscr{P}_{2}(\omega)=C(\omega)\left[\frac{\left|\mathscr{E}_{0}\right|^{2}\left[2\left|\mathscr{E}_{0}\right|^{2}-\left(y_{0}+y_{1}\right)\right]}{\left(\left|\mathscr{E}_{0}\right|^{2}-x_{1}\right)\left(\left|\mathscr{E}_{0}\right|^{2}-x_{2}\right)\left(\left|\mathscr{E}_{0}\right|^{2}-x_{3}\right)}\right. \\
&\left.\quad+\frac{\left(\left|\mathscr{E}_{0}\right|^{2}-y_{0}\right)\left(\left|\mathscr{E}_{0}\right|^{2}-y_{1}\right)}{\left(\left|\mathscr{E}_{0}\right|^{2}-x_{1}\right)\left(\left|\mathscr{E}_{0}\right|^{2}-x_{2}\right)\left(\left|\mathscr{E}_{0}\right|^{2}-x_{3}\right)}\left(1-\frac{1}{\left|\mathscr{E}_{0}\right|^{2}-x_{1}}+\frac{1}{\left|\mathscr{E}_{0}\right|^{2}-x_{2}}+\frac{1}{\left|\mathscr{E}_{0}\right|^{2}-x_{3}}\right]\right),
\end{aligned}
$$

and

$$
\begin{aligned}
\mathcal{P}_{3}(\omega)=C(\omega) \mathscr{E}_{0}^{2}[ & \frac{\left[2\left|\mathscr{E}_{0}\right|^{2}-\left(y_{0}+y_{1}\right)\right]}{\left(\left|\mathscr{E}_{0}\right|^{2}-x_{1}\right)\left(\left|\mathscr{E}_{0}\right|^{2}-x_{2}\right)\left(\left|\mathscr{E}_{0}\right|^{2}-x_{3}\right)} \\
& \left.-\frac{\left(\left|\mathscr{E}_{0}\right|^{2}-y_{0}\right)\left(\left|\mathscr{E}_{0}\right|^{2}-y_{1}\right)}{\left(\left|\mathscr{E}_{0}\right|^{2}-x_{1}\right)\left(\left|\mathscr{E}_{0}\right|^{2}-x_{2}\right)\left(\left|\mathscr{E}_{0}\right|^{2}-x_{3}\right)}\left[\frac{1}{\left|\mathscr{E}_{0}\right|^{2}-x_{1}}+\frac{1}{\left|\mathscr{E}_{0}\right|^{2}-x_{2}}+\frac{1}{\left|\mathscr{E}_{0}\right|^{2}-x_{3}}\right]\right) .
\end{aligned}
$$


This result constitutes the complete response of the medium for DFWM in the presence of arbitrary strong pumps but weak probe and signal beams. The quantities $\mathscr{P}_{1}(\omega)$ and $\mathcal{P}_{2}(\omega)$ correspond to the saturating absorption and nonlinear dispersion and $\mathcal{P}_{3}(\omega)$ is responsible for the phase-conjugate signal.

We can now introduce the polarization into the complete set of Maxwell equations to evaluate the response. The total field vector $\mathbf{E}$ satisfies the wave equation

$$
\nabla^{2} \mathbf{E}-\frac{1}{c^{2}} \frac{\partial^{2} \mathbf{E}}{\partial t^{2}}=\frac{1}{\epsilon_{0} c^{2}} N \frac{\partial^{2} \mathbf{P}}{\partial t^{2}},
$$

where $c$ and $\epsilon_{0}$ are respectively, the velocity of light and the dielectric constant in the vacuum, and $N$ the molecular density of the active species. In general, the coupledwave equations for the full set of waves must be solved. However, in the weak-probe-beam limit the equation for the pump fields can be decoupled from those of the probe and the signal fields. Moreover, in the slowly-varyingenvelope approximation, and neglecting the depletion of the pump waves, we find the standard coupled amplitude equations of motion considered by Abrams and Lind [8],

$$
\begin{aligned}
& \frac{\partial \mathscr{E}_{p}}{\partial z}=-\alpha \mathscr{E}_{p}-i \beta \mathscr{E}_{s}^{*}, \\
& \frac{\partial \mathscr{E}_{s}}{\partial z}=\alpha \mathscr{E}_{s}+i \beta \mathscr{E}_{p}^{*} .
\end{aligned}
$$

The quantities $\alpha$ and $\beta$ are deduced from the Fourier components of $\mathcal{P}_{2}(\omega)$ and $\mathcal{P}_{3}(\omega)$, respectively. This evaluation can be done analytically but the expressions are quite cumbersome and are not given here. For the solution of interest, the boundary conditions for Eqs. (3.13) are specified as

$$
\mathscr{E}_{p}(z=0)=\mathscr{E}_{p}(0), \quad \mathscr{E}_{s}(z=L)=0,
$$

$L$ being the length of the medium. Therefore the integration of Eqs. (3.13) yields the power-reflection coefficient

$R=\left|\frac{\mathscr{E}_{s}(0)}{\mathscr{E}_{p}(0)}\right|^{2}=\left|\frac{\beta \sin (\gamma L)}{\gamma \cos (\gamma L)+\alpha_{r} \sin (\gamma L)}\right|^{2}$,

where $\gamma^{2}=|\beta|^{2}-\alpha_{r}^{2}, \quad \alpha_{r}$ being the real part of the coefficient $\alpha$. The magnitude of $\gamma^{2}$ gives a measure of how the strength of the nonlinearity exceeds the absorption of the medium. If $|\beta|^{2} \geq \alpha_{r}^{2}$, the oscillation condition $R \rightarrow \infty$ becomes

$$
\tan (\gamma L)=-\frac{\gamma}{\alpha_{r}}
$$

For an appropriate length of the medium, this device acts as a reflection amplifier, since the reflected wave amplitude exceeds that of the input. The FWM process, in analogy to a backward parametric oscillator [40], can oscillate without mirror feedback.

\section{DISCUSSION}

All along, we assume that the medium is not excited at the initial time so that $w_{s}=-1$. In that case, to achieve large intensities of the phase-conjugate reflected wave, the nonlinear coupling must be sufficiently strong to dominate the absorption $\left(|\beta|>\alpha_{r}\right)$. If this threshold is exceeded, we can always find a sufficiently large value of $L$ inducing coupled-mode oscillations. To reach this threshold a minimal intensity is required and, of course, the experimental feasibility depends strongly on the magnitude of this intensity. Our purpose in this paper is to make clear that, in a number of situations, lower intensities are sufficient to reach high reflectivities by using the two-photon process of interest here, instead of the usual one-photon process.

\section{A. One-photon case}

In order to compare the one- and two-photon processes, we consider first the case where $d \neq 0$ and $\omega \simeq \omega_{21}$. For this resonant situation, no modifications have been observed with respect to the situation where $d=0$, even in the strong-field limit. According to the results obtained in the perturbative limit [37], this process is not very sensitive to the presence of the permanent dipole moments.

In the Appendix we recall briefly the Abrams and Lind results for the resonant case [8], in order to emphasize the highest reflectivity which can be achieved for a given intensity. In short, they have shown that for line-center operation, $\omega=\omega_{21}$, the reflectivity saturates but never becomes greater than unity. This saturation occurs because the increase in reflectivity with the medium length $L$ due to the increasing number of absorbers is compensated by the increase in absorption of the probe and conjugate signals as they propagate through the medium. To observe reflectivities higher than unity requires operation off line center. However, the cost of this increase of the powerreflection coefficient is the requirement that the pump intensity increases as $1+\delta^{2}$ times $I_{\text {sat }}^{0}$ (line-center saturation intensity). The minimal intensity needed to achieve coupled-mode oscillations is $I=2 I_{\text {sat }}^{0}$ when $\left|\omega-\omega_{21}\right| / \Gamma_{2}=\sqrt{3}$.

\section{B. Two-photon case}

Now we consider the two-photon case corresponding to the situation $\omega \simeq \omega_{21} / 2$. In the following, we normalize the intensities with respect to the line-center saturation intensity for the one-photon transition, that is to say,

$$
I_{\mathrm{sat}}^{0}=\frac{1}{2} \epsilon_{0} c \frac{\hbar^{2} \Gamma_{1} \Gamma_{2}}{\left|\mu_{12}\right|^{2}} .
$$

It is important to note that this saturation intensity depends on the longitudinal and transverse decay rates. Figure 3 shows the reflectivity for the one- and twophoton processes. Curve (a) is obtained from expression (A3) of the Appendix. It is the optimum value of the power-reflection coefficient which can be achieved for $I<I_{\text {sat }}^{0}$. Curve (b) results from Eq. (3.14) where $\alpha$ and $\beta$ have been deduced from Eqs. (3.11b) and (3.11c) by selecting the phase-matched components. Obviously, the two-photon process led to higher reflectivities than the one-photon process, for relatively low intensities. Note 


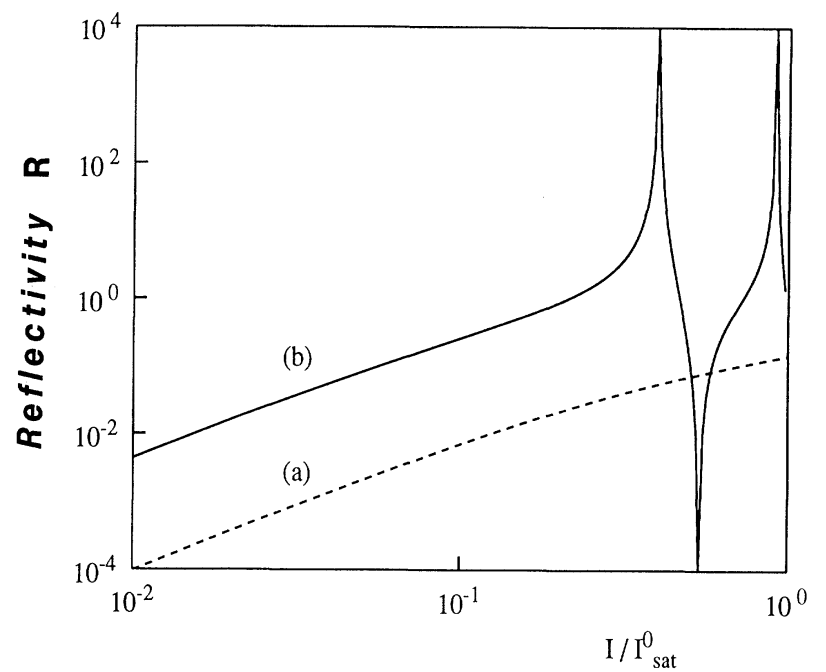

FIG. 3. Variation of the reflectivity $R$ vs $I / I_{\text {sat }}^{0}$, for $\Gamma_{1}=4 \times 10^{9} \quad \mathrm{~s}^{-1}, \quad \Gamma_{2}=2 \times 10^{9} \quad \mathrm{~s}^{-1}, \quad \mu_{12}=2.5 \times 10^{-29} \quad \mathrm{C} \mathrm{m}$, $d=10 \mu_{12}, \omega_{21}=4 \times 10^{15} \mathrm{~s}^{-1}$, and $N L=5 \times 10^{21} \mathrm{~mol} / \mathrm{cm}^{2}$, (a) one-photon transition case with $\delta=0$ and (b) two-photon transition case with $\delta^{\prime}=-1.5$.

that in curve (b) the normalized detuning

$$
\delta^{\prime}=\frac{\left(\omega-\omega_{21} / 2\right)}{\Gamma_{2}}
$$

is not equal to zero as will be discussed later. Figure 3 clearly illustrates the interest of this two-photon process for phase-conjugation experiments. In what follows, we are going to study the influence of the various parameters on the efficiency of this device.

First, let us look at the general behavior of the reflectivity as a function of the normalized intensity which is plotted in Fig. 4. Here $\delta^{\prime}$ is equal to zero. The

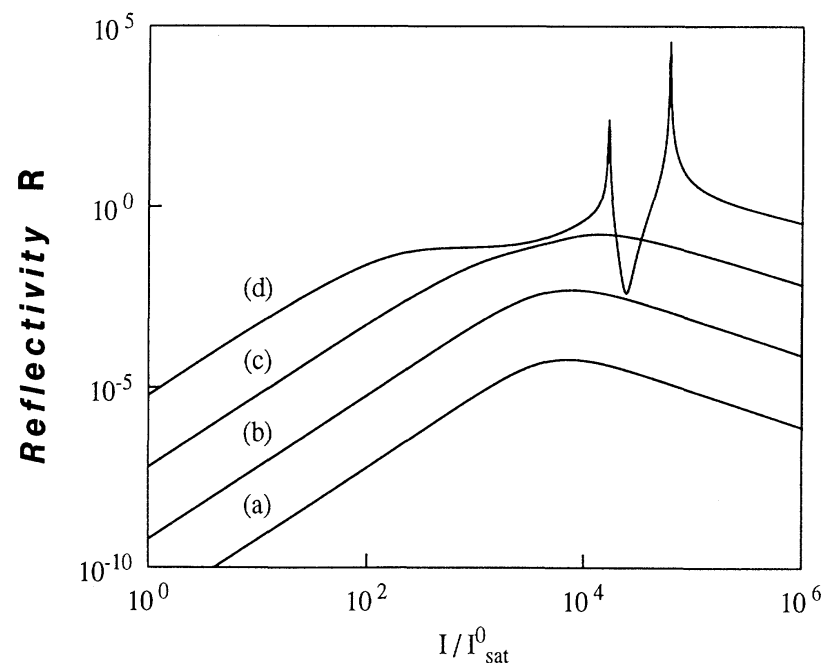

FIG. 4. Dependence of the reflectivity $R$ with $I / I_{\text {sat }}^{0}$ for the two-photon process. The values of the parameters are the same as in Fig. 3, except $\delta^{\prime}=0$ and (a) $N L=5 \times 10^{14}$, (b) $N L=5 \times 10^{15}$, (c) $N L=5 \times 10^{16}$, and (d) $N L=5 \times 10^{17} \mathrm{~mol} / \mathrm{cm}^{2}$. general behavior is similar to that of the resonant case. Actually, for small values of $N L$, the reflectivity increases linearly with $N L$. As $N L$ becomes large, the oscillation condition can be satisfied and amplification of the reflected wave is observed. The more $N L$ increases the more the intensity needed to observe amplification decreases and this continues up to the threshold value. Moreover, at low intensities, the power-reflection coefficient increases proportionally to $I^{2}$, then saturates before decreasing linearly with intensity. The saturation occurs for very high intensities here, $I_{\text {sat }}$ being about 10000 times the line-center saturation intensity. It corresponds to an intensity of about $0.2 \mathrm{MW} / \mathrm{cm}^{2}$ for the values of the parameters shown in Fig. 4. The need for high intensities occurs because the exciting fields are far from any dipole-allowed transition of the medium, so that coupling and absorption coefficients are much smaller than for the resonant case. The same reason holds to explain the need of longer samples to achieve high reflectivities.

As it can be seen in the perturbative limit [37], the two processes involved are quite different. First, in the resonant case, the second-order population is the basic quantity for the FWM signal. The perturbative chain can be schematized as follows:

$$
\rho_{11}^{(0)} \rightarrow\left[\begin{array}{l}
\rho_{21}^{(1)} \\
\rho_{12}^{(1)}
\end{array}\right] \rightarrow\left[\begin{array}{l}
\rho_{11}^{(2)} \\
\rho_{22}^{(2)}
\end{array}\right] \rightarrow\left[\begin{array}{l}
\rho_{21}^{(3)} \\
\rho_{12}^{(3)}
\end{array}\right)
$$

For the two-photon process involved here, it has been shown [37] that the main contribution to the DFWM signal can be represented by two perturbation chains,

$$
\begin{aligned}
& \rho_{11}^{(0)} \rightarrow\left[\begin{array}{l}
\rho_{21}^{(1)} \\
\rho_{12}^{(1)}
\end{array}\right] \rightarrow\left[\begin{array}{l}
\rho_{21}^{(2)} \\
\rho_{12}^{(2)}
\end{array}\right] \rightarrow\left[\begin{array}{c}
\rho_{21}^{(3)} \\
\rho_{12}^{(3)}
\end{array}\right], \\
& \rho_{11}^{(0)} \rightarrow\left[\begin{array}{l}
\rho_{21}^{(1)} \\
\rho_{12}^{(1)}
\end{array}\right] \rightarrow\left[\begin{array}{l}
\rho_{21}^{(2)} \\
\rho_{12}^{(2)}
\end{array}\right] \rightarrow\left[\begin{array}{c}
\rho_{22}^{(3)} \\
\rho_{11}^{(3)}
\end{array}\right] .
\end{aligned}
$$

Unlike the case of two-level phase conjugation, no population grating is involved in this process. Therefore the coherences are the basic quantities.

From the previous discussion it has to be mentioned that we recover here a situation similar to the one observed in standard two-photon DFWM [18] where at least there levels are involved. At low intensities, the primary phase-conjugate contribution results from the conjugated signal interacting with a two-photon coherence term whose pump wave-vector dependence cancels out. The perturbation chains can be schematized as follows:

$$
\begin{aligned}
& \rho_{11}^{(0)} \rightarrow\left[\begin{array}{l}
\rho_{21}^{(1)} \\
\rho_{12}^{(1)}
\end{array}\right] \rightarrow\left[\begin{array}{l}
\rho_{13}^{(2)} \\
\rho_{31}^{(2)}
\end{array}\right] \rightarrow\left[\begin{array}{l}
\rho_{23}^{(3)} \\
\rho_{32}^{(3)}
\end{array}\right], \\
& \rho_{11}^{(0)} \rightarrow\left[\begin{array}{c}
\rho_{21}^{(1)} \\
\rho_{12}^{(1)}
\end{array}\right] \rightarrow\left[\begin{array}{l}
\rho_{13}^{(2)} \\
\rho_{31}^{(2)}
\end{array}\right] \rightarrow\left[\begin{array}{l}
\rho_{21}^{(3)} \\
\rho_{12}^{(3)}
\end{array}\right] .
\end{aligned}
$$

A comparison between chains (4.3) and (4.5), (4.4) and (4.6), shows that owing to a difference in permanent dipole moments between the two levels, the excited state acts as the intermediate states in a standard two-photon 
process. Here, $d=\mu_{22}-\mu_{11}$ takes the role of the transition moment between the intermediate state and the final state, $\mu_{23}$. However, in perturbation chain (4.4), thirdorder oscillating populations play the role of the coherences in path (4.5). Because the diagonal elements appear directly in the expression for the polarization as shown in Eq. (2.11), oscillating populations take part to the phaseconjugated signal.

For sufficiently large pump intensities, the two processes behave quite differently. In the theory of two-photon phase conjugation described by $\mathrm{Fu}$ and Sargent [18], the Stark-shifted line center prevents bleaching and leads to an intensity-independent reflectivity at large intensities. In this limit, a pure-index phase-conjugation contribution interferes with the index contribution due to the excitation of a two-photon coherence by the pump waves. The first contribution arises from the formation of a spatial grating and is the strong-field analog to a perturbation path like the one labeled (4.2). The second term is the strong-field analog to perturbation chains (4.5) and (4.6). This intensity-independent reflectivity contrasts with the decrease of $R$ depicted in Fig. 4. Here, the Stark-shifted line center cannot prevent bleaching. We have numerically tested the contribution which results from offresonant single-photon transition by taking $d=0$. It appears that this contribution is negligible, so that no spatial grating term yields phase conjugation. We have also tested the respective contributions of $w_{1}$ and $\rho_{1}$. These two contributions behave similarly and have approximately the same magnitude. For this situation where $\Gamma_{2}=\Gamma_{1} / 2$ and $\delta^{\prime}=0$, populations and coherences contribute in the same ratio. Finally, we can note that the saturation intensity seems to behave proportionally to $\sqrt{\Gamma_{1} \Gamma_{2}}$ as does the standard two-photon case, whereas the threshold intensity appears to behave proportionally to $\Gamma_{1} \Gamma_{2}$.

Now, we are interested in the influence of the various parameters on the self-oscillation threshold intensity, in order to deduce criteria to achieve high reflectivities with this two-photon process. Figure 5 shows the powerreflection coefficient as a function of detuning $\delta^{\prime}$. The intensity is equal to the line-center saturation intensity $I_{\text {sat }}^{0}$. Obviously, far from resonance oscillation occurs at smaller medium length than at exact half resonance. By studying $|\beta|-\alpha_{r}$ as a function of $\delta^{\prime}$ and intensity, it appears that the threshold is considerably reduced when using frequencies which differ from exact half resonance by an amount of a few $\Gamma_{2}$. This arises because the decrease of the absorption is much faster than the one of the nonlinear coupling coefficient when the detuning increases, so that absorption can be dominated. Furthermore, for intensities below the saturation intensity $I_{\text {sat }}$, the reflectivity behaves symmetrically with respect to the half resonance. When the pump intensity is increased, this behavior becomes asymmetric and much higher powerreflection coefficients can be achieved for negative detunings than for positive ones. This shift of the reflectivity occurs consequently to the one of the absorption and the nonlinear coupling coefficient. In this limit, we obtain a region with gain $\left(\alpha_{r}<0\right)$. To get a better knowledge of such systems under strong excitation, a complete

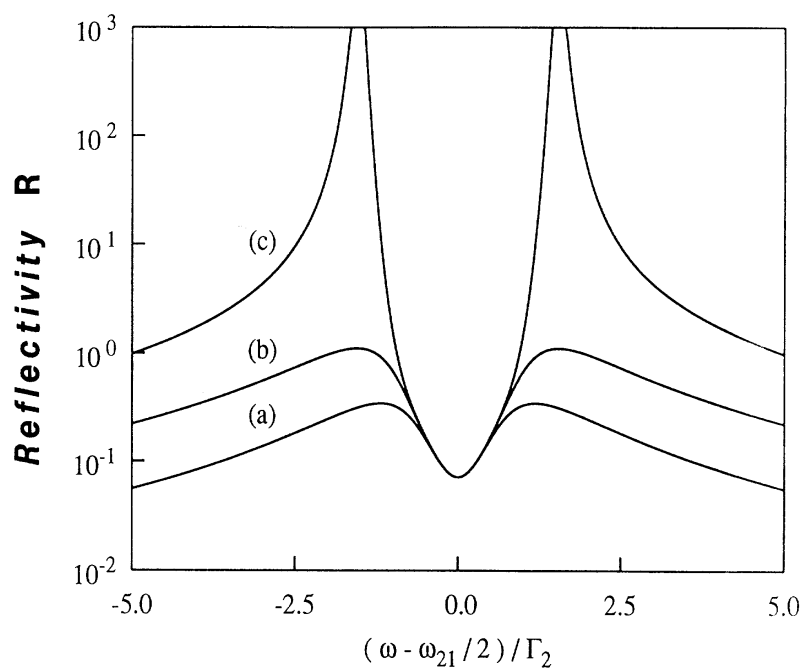

FIG. 5. Dependence of the reflectivity $R$ with normalized detuning, $\delta^{\prime}=\left(\omega-\omega_{21} / 2\right) / \Gamma_{2}$ for the two-photon process. The values of the parameters are the same as in Fig. 3, expect $I=I_{\text {sat }}^{0}$ and (a) $N L=5 \times 10^{20}$, (b) $N L=10^{21}$, and (c) $N L=1.9 \times 10^{21}$ $\mathrm{mol} / \mathrm{cm}^{2}$.

quantum-mechanical treatment, such as the dressed-atom approach [41], is necessary. Moreover, here all the waves have the same frequency and this makes the understanding of the dynamical Stark effect difficult. At least, the probe should be nondegenerate with the pump waves [42-45].

In order to study the influence of pure dephasing on the normalized threshold intensity, $I_{\text {thres }}$, we have plotted $|\beta|-\alpha_{r}$ as a function of normalized intensity in Fig. 6. It is important to note that the normalization factor $I_{\text {sat }}^{0}$ is proportional to the dephasing rate. We introduce this

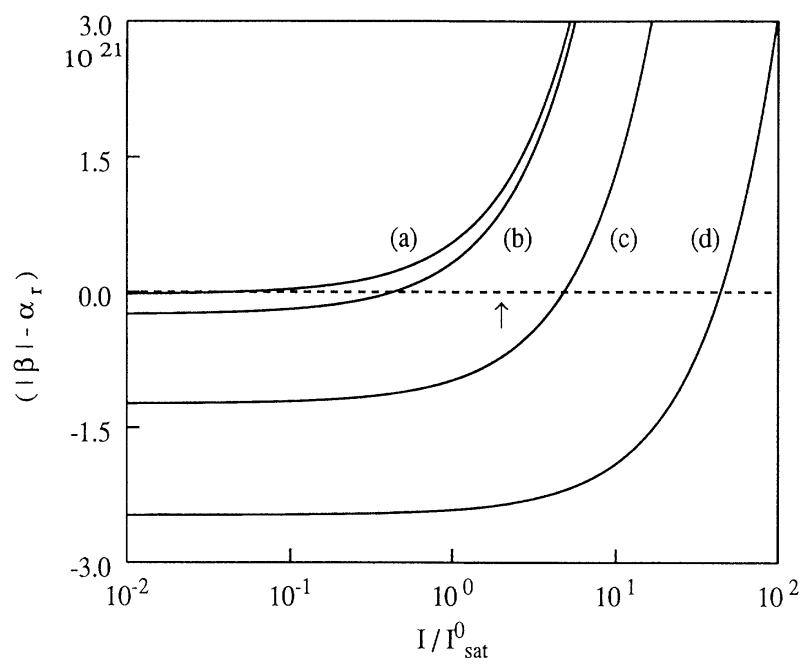

FIG. 6. We represent $|\beta|-\alpha_{r}$ as a function of $I / I_{\text {sat }}^{0}$ for the two-photon process. The values of the parameters are the same as in Fig. 3, except (a) $\Gamma_{2}=2 \times 10^{9}$, (b) $\Gamma_{2}=2 \times 10^{10}$, (c) $\Gamma_{2}=10^{11}$, and (d) $\Gamma_{2}=2 \times 10^{11} \mathrm{~s}^{-1}$. 


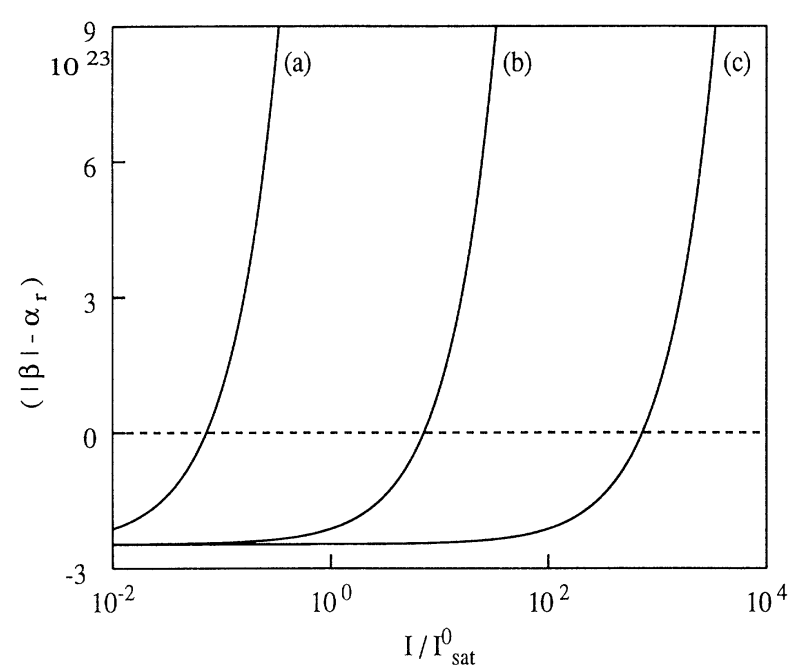

FIG. 7. We represent the same variations as in Fig. 6 except that here $\delta^{\prime}=-6, \Gamma_{2}=2 \times 10^{9}$, and (a) $d=10 \mu_{12}$, (b) $d=\mu_{12}$, and (c) $d=0.1 \mu_{12}$.

normalization because our purpose is to make a comparison with the resonant process. Figure 6 shows that $I_{\text {thres }}$ increases strongly with pure dephasing. The arrow denotes the normalized threshold intensity for the resonant case, where $I_{\text {sat }}^{0}$ takes into account the sole dependence on the dephasing rate. The oscillation conditions are not easily achieved because the off-resonant singlephoton contribution increases as well. In fact, we have studied the behavior of $\alpha_{r}$ and $|\beta|$ as a function of detuning when the dephasing becomes important, taking $d=10 \mu_{12}$ and $d=0$. For $d=10 \mu_{12}$ the spectrum gets broader, and the broadening is proportional to the dephasing rate, as expected. In addition, we observe a constant contribution in this spectral range. We recover this contribution by taking $d=0$. Therefore it results from off-resonance single-photon processes. For the dephasing rates given in Fig. 6, this background is much more important for the absorption than for the nonlinear coupling, and consequently prevents more and more $|\beta|$ from dominating $\alpha_{r}$.

Finally, in Fig. 7 we have plotted $|\beta|-\alpha_{r}$ as a function of the intensity for various values of $d$. Note that the normalized detuning is $\delta^{\prime}=-6$. We recover the situation obtained in the perturbative limit [37], that is to say, the strong dependence of this two-photon process with the difference of the permanent dipole moments of the two levels. It seems that $I_{\text {thres }}$ but also $I_{\text {sat }}$ behave proportionally to $d^{-2}$. For intensities of the order of $I_{\text {sat }}^{0}$, the absorption coefficient is almost independent of $d$, even if $|\beta|$ increase as $d^{2}$. Therefore the greater the difference in dipole moments, the smaller the normalized threshold intensity for this near-half-resonant case.

\section{CONCLUSION}

We have developed a general theory of two-photon DFWM phase conjugation for a medium modeled by homogeneously broadened two-level systems having un- equal permanent dipole moments, and valid for arbitrarily strong excitation fields. In the particular case of weak probe and signal beams, the reflectivity of the phaseconjugated wave has been evaluated. Comparisons between this two-photon process and the well-known oneand standard two-photon processes have been established. We have discussed the influence of frequency detuning, dephasing rate, and permanent dipole moments on the power-reflection coefficient, especially near the threshold intensity for which coupled-mode oscillations are predicted.

For polar molecules, it has been clearly demonstrated by using field frequencies in the vicinity of the half transition frequency that it must be possible to obtain much higher reflectivities than in the near-resonance case. In this study, we have established a criterion to define the interesting physical situations. In fact, because the absorption decreases faster than the nonlinear coupling when deviating from half resonance, it is of interest to consider situations where detunings from half resonance are of the order of a few dephasing rates $\left(\left|\omega-\omega_{21} / 2\right| \sim \Gamma_{2}\right)$. Furthermore, pure dephasing must be small $\left(\Gamma_{2} \leqslant 10 \Gamma_{1}\right)$, in order to avoid off-resonance single-photon contributions which increase the absorption more rapidly than the nonlinear coupling. Finally, for intensities comparable to the line-center saturation intensity $I_{\text {sat }}^{0}$, the absorption is almost independent of $d$ whereas nonlinear coupling increases like $d^{2}$. Consequently, the more $d=\mu_{22}-\mu_{11}$ is important with respect to $\mu_{12}$, the more this device will be adapted to phaseconjugation experiments.

To conclude, it has to be noted that this two-photon process occurs on a spectral range where the medium is transparent. This can be very useful in phase-conjugation experiments where thermal gratings and sample degradation should be avoided. Finally, in the region where high-power-reflection coefficients are predicted, the absorption and depletion of the pump waves can no longer be neglected [10]. A complete account of these phenomena requires the solution of more complex dynamical equations for the field amplitudes and their corresponding phases. They will be discussed in a forthcoming presentation.

\section{ACKNOWLEDGMENT}

Institut de Physique et Chimie des Matériaux de Strasbourg is "Unite Mixte No. 380046 du Centre National de la Recherche Scientifque, de l'Université Louis Pasteur et de l'Ecole des Hautes Etudes des Industries Chimiques de Strasbourg."

\section{APPENDIX}

In this appendix we briefly recall the results obtained by Abrams and Lind for resonant DFWM phase conjugation [8], in order to specify the limiting value of the power-reflection coefficient for the one-photon resonant case. If the two pump waves have the same intensity, the expressions for $\alpha$ and $\beta$ are given by 


$$
\begin{aligned}
& \alpha=\alpha_{0} \frac{1-i \delta}{1+\delta^{2}} \frac{1+2 I / I_{\mathrm{sat}}}{\left(1+4 I / I_{\mathrm{sat}}\right)^{3 / 2}}=\alpha_{r}-i \alpha_{i}, \\
& \beta=i \alpha_{0} \frac{1-i \delta}{1+\delta^{2}} \frac{2 I / I_{\mathrm{sat}}}{\left(1+4 I / I_{\mathrm{sat}}\right)^{3 / 2}} .
\end{aligned}
$$

Here $\alpha_{0}$ stands for the line-center small-signal-field attenuation coefficient, $\delta$ is the normalized detuning from the line center defined by $\delta=\left(\omega-\omega_{21}\right) / \Gamma_{2}$, and $I_{\text {sat }}=\left(1+\delta^{2}\right) I_{\text {sat }}^{0}$ where $I_{\text {sat }}^{0}$ is the line-center saturation intensity. We shall be interested in the optimization of the conjugate signal. From the behavior of $|\beta|^{2}-\alpha_{r}^{2}$ one can see that the smallest intensity needed to reach the oscillation conditions is achieved at $I / I_{\text {sat }}^{0}=2$ for $\delta=\sqrt{3}$. Furthermore, for $I<I_{\text {sat }}^{0}$ no oscillation can be reached. In this case, the optimum value of the power-reflection coefficient is obtained for $\delta=0$,

$$
R_{\mathrm{opt}}=\left(\frac{2 I / I_{\mathrm{sat}}^{0}}{1+2 I / I_{\mathrm{sat}}^{0}+\sqrt{1+4 I / I_{\mathrm{sat}}^{0}}}\right)^{2}
$$

[1] For a review see, for example, Optical Phase Conjugation, edited by R. A. Fisher (Academic, New York, 1983); B. Ya. Zel'dovich, N. F. Pilipetsky, and V. V. Shkunov, Principles of Phase Conjugation (Springer-Verlag, Berlin, 1985).

[2] A. Yariv and D. M. Pepper, Opt. Lett. 1, 16 (1977).

[3] A. Yariv, Opt. Lett. 16, 1376 (1991).

[4] D. E. Watkins, J. F. Figueira, and S. J. Thomas, Opt. Lett. 5, 169 (1980).

[5] I. C. Khoo, Appl. Phys. Lett. 47, 908 (1985).

[6] L. Richard, J. Maurin, and J. P. Huignard, Opt. Commun. 57, 365 (1986).

[7] W. M. Dennis and W. Blau, Opt. Commun. 86, 371 (1986).

[8] R. L. Abrams and R. C. Lind, Opt. Lett. 2, 94 (1978); 3, 205(E) (1978); see also Optical Phase Conjugation, edited by R. A. Fisher (Academic, New York, 1983), p. 211.

[9] T. Fu and M. Sargent III, Opt. Lett. 4, 366 (1979).

[10] W. P. Brown, J. Opt. Soc. Am. B 73, 629 (1983).

[11] D. Bloch and M. Ducloy, J. Opt. Soc. Am. B 73, 635 (1983); 73, 1844(E) (1983).

[12] A. A. Villaeys and J. P. Lavoine, Opt. Commun. 63, 349 (1987).

[13] J. P. Lavoine and A. A. Villaeys, Opt. Commun. 59, 160 (1986).

[14] H. Fujiwara and K. Nakagawa, J. Opt. Soc. Am. B 4, 121 (1987).

[15] H. Nakano and J. Sakai, IEEE J. Quantum Electron. 27, 2495 (1991).

[16] M. Matsuoka, Opt. Commun. 15, 84 (1975).

[17] D. C. Haueisen, Opt. Commun. 28, 183 (1979).

[18] T. Fu and M. Sargent III, Opt. Lett. 5, 433 (1980).

[19] M. Sargent III, S. Ovadia, and M. Hong Lu, Phys. Rev. A 32, 1596 (1985).

[20] M. Kauranen, D. J. Gauthier, M. S. Malcuit, and R. W. Boyd, Phys. Rev. A 40, 1908 (1989).

[21] B. Dick and G. Hohlneicher, J. Chem. Phys. 76, 5755 (1982).

[22] G. F. Thomas and W. J. Meath, Mol. Phys. 46, 743 (1982); 48, 649(E) (1983).
[23] B. E. Scharf and Y. B. Band, Chem. Phys. Lett. 144, 165 (1988).

[24] Y. B. Band, R. Bavli, and D. F. Heller, Chem. Phys. Lett. 156, 405 (1989).

[25] R. Bavli and Y. B. Band, Phys. Rev. A 43, 507 (1991); 43, 5039 (1991).

[26] R. Bavli, D. F. Heller, and Y. B. Band, Phys. Rev. A 41, 3960 (1990).

[27] T. Kobayashi, N. C. Kothari, and H. Uchiki, Phys. Rev. A 29, 2727 (1984).

[28] N. C. Kothari and T. Kobayashi, IEEE J. Quantum Electron. QE-20, 418 (1984).

[29] W. J. Meath and E. A. Power, J. Phys. B 17, 763 (1984).

[30] W. J. Meath and E. A. Power, Mol. Phys. 51, 585 (1984).

[31] M. A. Kmectic and W. J. Meath, Phys. Lett. 108A, 340 (1985).

[32] M. A. Kmectic, R. A. Thuraisingham, and W. J. Meath, Phys. Rev. A 33, 1688 (1986).

[33] G. F. Thomas, Phys. Rev. A 33, 1033 (1986).

[34] M. A. Kmectic and W. J. Meath, Phys. Rev. A 41, 1556 (1990).

[35] G. F. Thomas, J. Chem. Phys. 94, 1667 (1991).

[36] A. E. Kondo and W. J. Meath, Mol. Phys. 74, 113 (1991).

[37] J. P. Lavoine, C. Hoerner, and A. A. Villaeys, Phys. Rev. A 44, 5947 (1991).

[38] M. A. Antón and I. Gonzalo, J. Opt. Soc. Am. B 8, 1035 (1991).

[39] R. C. Lind, D. G. Steel, and G. J. Dunning, Opt. Eng. 21, 190 (1982).

[40] S. E. Harris, Appl. Phys. Lett. 9, 114 (1966).

[41] C. Cohen-Tannoudji and S. Reynaud, J. Phys. B 10, 345 (1977); 10, 365 (1977).

[42] B. R. Mollow, Phys. Rev. 188, 1969 (1969).

[43] B. R. Mollow, Phys. Rev. A 5, 2217 (1972).

[44] R. W. Boyd, M. G. Raymer, P. Narum, and D. J. Harter, Phys. Rev. A 24, 411 (1981).

[45] D. G. Steel and R. C. Lind, Opt. Lett. 6, 587 (1981). 